\title{
Quantum lithography with classical light: Generation of arbitrary patterns
}

\author{
Qingqing Sun, ${ }^{1,2}$ Philip R. Hemmer, ${ }^{3}$ and M. Suhail Zubairy ${ }^{1,2}$ \\ ${ }^{1}$ Department of Physics and Institute of Quantum Studies, Texas A\&M University, College Station, Texas 77843-4242, USA \\ ${ }^{2}$ Texas A\&M University at Qatar, Education City, P.O. Box 23874, Doha, Qatar \\ ${ }^{3}$ Department of Electrical and Computer Engineering, Texas A\&M University, College Station, Texas 77843-3128, USA
}

(Received 6 June 2006; revised manuscript received 20 March 2007; published 27 June 2007)

\begin{abstract}
Based on our previous scheme [Hemmer et al., Phys. Rev. Lett. 96, 163603 (2006)] we show the procedures to obtain any one- or two-dimensional pattern by multiple exposures. A key modification to the resonance condition is made to achieve subwavelength pattern resolution. The level separation of the substrate does not pose an upper limit to the frequency summation. So the fundamental frequency can be high and the number of Fourier terms can be large, making our scheme very suitable for subwavelength arbitrary patterns.
\end{abstract}

DOI: 10.1103/PhysRevA.75.065803

PACS number(s): 42.50.St, $42.50 . \mathrm{Hz}, 85.40 . \mathrm{Hp}$

\section{INTRODUCTION}

Optical lithography is widely used in semiconductor device fabrication to write a pattern on a substrate. When exposed to the control light, the photoresist layer coated on the substrate is hardened to form a pattern. After etching, the photoresist layer is removed, leaving the desired pattern printed on the substrate. However, the highest resolution one can achieve with classical uncorrelated light is of half the wavelength [1]. This is due to the fundamental limit of diffraction, the so-called Rayleigh criterion [2].

The semiconductor industry is always demanding higher resolution. Current wavelengths in use have gone into the deep ultraviolet region [3]. Under this situation, many schemes have been proposed to beat the diffraction limit [4-7]. A potential scheme that generated a great deal of interest is quantum lithography [6], in which an entangled $N$-photon state $\left(|0\rangle|N\rangle+e^{i N \varphi}|N\rangle|0\rangle\right) / \sqrt{2}$ illuminates an $\mathrm{N}$-photon absorbing substrate. Since these photons are correlated, any phase difference along the path will be magnified by a factor of $N$. Therefore the interference fringes on the substrate become closer to each other and the maximum resolution is now $\lambda / 2 N$, which is far below the Rayleigh criterion. A proof-of-principle experiment has been done based on entangled photon pairs [8]. In spite of the formal simplicity, this method is not easy to realize due to the following reasons. (a) Preparing and keeping a high order entangled Fock state is quite difficult in experiment. (b) The limited photon number makes the exposure very slow. These practical difficulties have stimulated some alternative methods based on classical fields [9-11].

In Ref. [12], a novel approach was proposed to implement quantum lithography using the classical light. This is accomplished by correlating wave vector and frequency in a narrow band multiphoton detection process, so that the subwavelength fringes can be obtained. This method has the advantages of high efficiency, visibility, and spatial coherence. However, a concern is expressed that this scheme can generate only a tight pattern of parallel lines [13]. In this Brief Report we show the procedures to obtain arbitrary patterns in both one and two dimensions. It is done by multiple exposures which correspond to a truncated Fourier series. The modification of the resonance condition allows us to choose a fundamental frequency much larger than the signal frequencies, which enables subwavelength resolution. A unique property of our scheme is that there is no frequency upper limit due to the level separation of the substrate. The possibility of large fundamental frequency and many Fourier components makes our scheme very suitable to fabricate an arbitrary pattern at the subwavelength scale.

\section{QUANTUM LITHOGRAPHY WITH CLASSICAL LIGHT}

First we briefly review the method in our previous paper [12]. The scheme is illustrated in Fig. 1. Two bunches of

(a)

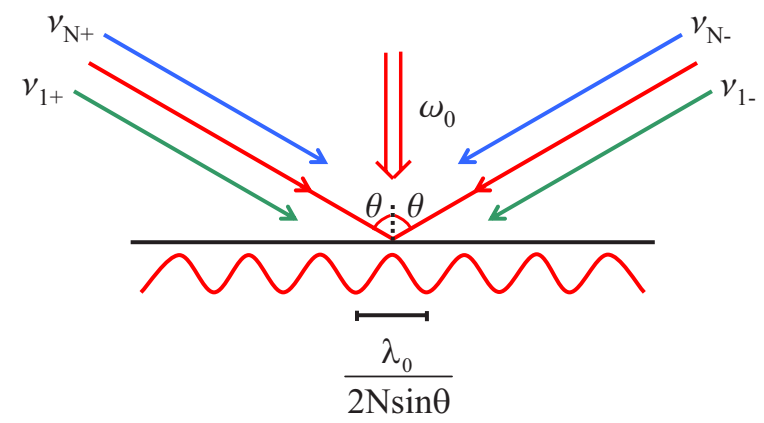

(b)

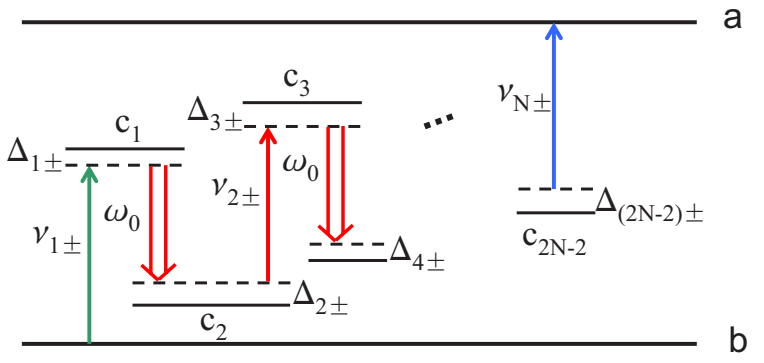

FIG. 1. (Color online) (a) The scheme of interferometric lithography. Two bunches of signal fields counterpropagate $(\theta=\pi / 2)$ and the drive field incidents normally. (b) The level structure of the substrate atom. Either bunch of fields together with the drive field satisfies the multiphoton resonance. $\Delta_{n \pm}$ is the detuning of intermediate level $c_{n}$. 
signal fields counterpropagate along the substrate $(\theta=\pi / 2)$ and a drive field is incident normally. Either bunch of fields together with the drive field can excite the multiphoton process from level $|b\rangle$ to $|a\rangle$. The photons from the signal fields of frequencies $\nu_{n \pm}$ are absorbed and the photons of the drive field with frequency $\omega_{0}$ are emitted. The $N$ signal photons satisfy a frequency summation resonance

$$
\sum_{n=1}^{N} \nu_{n \pm}=\omega_{a b}+(N-1) \omega_{0}=N \nu_{0},
$$

so that the $N$-photon wave vector, $N \nu_{0} / c=2 \pi /\left(\lambda_{0} / N\right)$, is the same for both bunches. We further require that any interchange of photons between bunches, $\nu_{n+} \leftrightarrow \nu_{n^{\prime}-}$, results in a loss of resonance. Therefore only two resonant processes make up the interference.

The electric field on the surface is

$$
\begin{aligned}
E(x, t)= & \sum_{n=1}^{N}\left[\mathcal{E}_{S_{n+}} e^{i\left(k_{n+} x-\nu_{n+} t\right)}+\mathcal{E}_{S_{n-}} e^{i\left(k_{n-} x-\nu_{n-} t\right)}\right] \\
& +\mathcal{E}_{D} e^{-i \omega_{0} t}+\text { c.c. },
\end{aligned}
$$

where $k_{n \pm}= \pm\left(\nu_{n \pm} / c\right)$. In the level structure of the substrate, the intermediate levels $c_{j}$ are off-resonant by detunings $\Delta_{1 \pm}$ $=\omega_{c_{1} b}-\nu_{1 \pm}, \Delta_{2 \pm}=\nu_{1 \pm}-\omega_{0}-\omega_{c_{2} b}, \ldots, \Delta_{(2 N-2) \pm}=\omega_{a c_{2 N-2}}-\nu_{N \pm}$.

Under the conditions of multiphoton resonance, the leading contributions to the multiphoton excitation rate come from the two resonant processes, i.e.,

$$
R^{(2 N-1)}(x, t) \propto \frac{d}{d t}\left|e^{i\left(N \nu_{0} x / c\right)} r_{+}^{(2 N-1)}(t)+e^{-i\left(N \nu_{0} x / c\right)} r_{-}^{(2 N-1)}(t)\right|^{2} .
$$

If the one-photon detunings are large and $\mathcal{E}_{S_{n \pm}}$ are suitable, the excitation amplitudes $r_{ \pm}(t)$ could be approximately equal with a phase difference. Factoring them out we find that the remaining expression looks like the interference of single photon absorption with $k=N \nu_{0} / c$. So the exposure patterns are fringes with distance $\lambda_{0} / 2 N$.

\section{ARBITRARY ONE-DIMENSIONAL PATTERN}

Once parallel fringes are obtained, Fourier theory tells us that one can compose any pattern from a set of fringes. However, a careful look at Eq. (1) shows that the fundamental frequency $\nu_{0}$ is the average of $\nu_{n \pm}$. So the fringes can be subwavelength, but the pattern resolution as decided by the fundamental frequency is not. $\nu_{0}$ is also limited by the level separation $\omega_{a b}$ like the other schemes.

To remove these limitations, we change the resonance condition into

$$
\sum_{n=1}^{n_{N}} \nu_{n \pm}=\omega_{a b}+\left(n_{N}-1\right) \omega_{N}=N \nu_{0} .
$$

This is the key equation. Note the main difference is that the number of signal fields involved in a multiphoton resonance changes from $N$ into $n_{N}$. As far as the frequency summation equals $N \nu_{0}$, there is no requirement this process must absorb
$N$ photons. Now the fundamental frequency $\nu_{0}$ can be much larger than $\nu_{n \pm}$ to obtain a subwavelength resolution. $\nu_{0}$ can even be much larger than $\omega_{a b}$, which is a unique property due to the Doppleron-type resonances of our scheme. The drive frequency is also changed from $\omega_{0}$ to $\omega_{N}$, which can be different for each $N$. The arbitrary photon number $n_{N}$ and drive frequency $\omega_{N}$ give us more freedom to choose the fields.

To show the procedures toward an arbitrary onedimensional (1D) pattern, we consider a high frequency $\nu_{0}$. For any $N(=1,2,3, \ldots)$ we can always find some suitable $n_{N}$ and $\omega_{N}$ to achieve the multiphoton wave vector $N \nu_{0} / c$. Two bunches of signal fields grazing from $+x$ and $-x$ directions will give the excitation rate

$$
R^{\left(2 n_{N}-1\right)}(x, t) \propto \frac{d}{d t}\left|e^{i\left(N \nu_{0} x / c\right)} r_{+}^{\left(2 n_{N}-1\right)}(t)+e^{-i\left(N \nu_{0} x / c\right)} r_{-}^{\left(2 n_{N}-1\right)}(t)\right|^{2} .
$$

For each $N$ we can use the above method to make an exposure. After multiple exposures we get fringes corresponding to $N=1,2,3, \ldots, N_{\max }$. The final pattern is

$$
\begin{aligned}
P(x) & =\sum_{N=1}^{N_{\max }} \int_{0}^{t_{N}} R^{\left(2 n_{N}-1\right)}(x, t) d t \\
& =\sum_{N=1}^{N_{\max }} c_{N}\left|r^{\left(2 n_{N}-1\right)}\left(t_{N}\right)\right|^{2} \mid e^{i\left(N \nu_{0} x / c\right)}+e^{-i\left(N \nu_{0} x / c\right)} e^{\left.i \theta_{N}\right|^{2}} \\
& =Q+\sum_{N=0}^{N_{\max }}\left(a_{N} \cos \frac{2 N \nu_{0} x}{c}+b_{N} \sin \frac{2 N \nu_{0} x}{c}\right),
\end{aligned}
$$

where $c_{N}$ is the ratio coefficient in $R^{\left(2 n_{N}-1\right)}$. Here $P(x)$ is a truncated Fourier series with a penalty deposition $Q$. Such a series can approximate any 1D pattern if enough components are included. The coefficients and phases of each component can be controlled by $\mathcal{E}_{S_{n \pm}}$ and $t_{N}$.

\section{ARBITRARY TWO-DIMENSIONAL PATTERN}

For practical purposes, we would like to see how this method can be applied to generate an arbitrary pattern in two dimensions. Such a generalization still relies on multiple exposures, using two bunches of signal fields each time. However, the direction of these two bunches is not limited to the $x$ axis.

First send in the two bunches from the opposite directions of $\pm(N \hat{x}+M \hat{y}) / \sqrt{N^{2}+M^{2}}$ and make an exposure. The sum frequency of either bunch should satisfy

$$
\sum_{n=1}^{\sqrt[n]{N^{2}+M^{2}}} \nu_{n \pm}=\sqrt{N^{2}+M^{2}} \nu_{0} .
$$

Like the 1D case, we have the multiphoton excitation rate

$$
\begin{aligned}
R^{\left(2 n \sqrt{N^{2}+M^{2}}-1\right)}(x, t) \propto & \frac{d}{d t} \mid e^{i\left[\left(N \nu_{0} x+M \nu_{0} y\right) / c\right]} r_{+}^{(2 n-1)}(t) \\
& +\left.e^{-i\left[\left(N \nu_{0} x+M \nu_{0} y\right) / c\right]} r_{-}^{(2 n-1)}(t)\right|^{2} .
\end{aligned}
$$

Then change the directions of these two bunches into $\pm(N \hat{x}$ 
$-M \hat{y}) / \sqrt{N^{2}+M^{2}}$ and make another exposure. Note the field coefficients and phases for the two exposures could be different. For each nonzero $(N, M)$ pair that satisfies $\sqrt{N^{2}+M^{2}}$ $\leqslant N_{\max }$ we make two exposures like this. If $N$ or $M=0$ then only one exposure is needed. From Eq. (6) we find the final exposure pattern,

$$
\begin{aligned}
P(x, y)= & \sum_{0<\sqrt{N^{2}+M^{2}} \leqslant N_{\max }}\left[a_{N M} \cos \frac{2 \nu_{0}(N x+M y)}{c}+b_{N M} \sin \frac{2 \nu_{0}(N x+M y)}{c}+\sqrt{a_{N M}^{2}+b_{N M}^{2}}\right. \\
& \left.+c_{N M} \cos \frac{2 \nu_{0}(N x-M y)}{c}+d_{N M} \sin \frac{2 \nu_{0}(N x-M y)}{c}+\sqrt{c_{N M}^{2}+d_{N M}^{2}}\right] \\
= & Q+\sum_{0 \leqslant \sqrt{N^{2}+M^{2}} \leqslant N_{\max }}\left[\left(a_{N M}+c_{N M}\right) \cos \frac{2 \nu_{0} N x}{c} \cos \frac{2 \nu_{0} M y}{c}+\left(-a_{N M}+c_{N M}\right) \sin \frac{2 \nu_{0} N x}{c} \sin \frac{2 \nu_{0} M y}{c}\right. \\
& \left.+\left(b_{N M}+d_{N M}\right) \sin \frac{2 \nu_{0} N x}{c} \cos \frac{2 \nu_{0} M y}{c}+\left(b_{N M}-d_{N M}\right) \cos \frac{2 \nu_{0} N x}{c} \sin \frac{2 \nu_{0} M y}{c}\right] .
\end{aligned}
$$

Again this is a truncated 2D Fourier series with a penalty deposition. It can approximate any two-dimensional pattern in principle.

As an example, we consider the test function

$$
F(x, y)=\left\{\begin{array}{l}
h \text { if }-\frac{\pi}{2}<\frac{2 \nu_{0} x}{c}, \frac{2 \nu_{0} y}{c}<\frac{\pi}{2} \\
0 \text { elsewhere. }
\end{array}\right.
$$

For the upper limit $N_{\max }=10$ we get the truncated Fourier series $P(x)-Q$ as shown in Fig. 2. Except for the abrupt ramp and the corners of the hat, the error to the test function is within $\pm 0.1 \mathrm{~h}$, which is acceptable considering the number of components included. The penalty deposition $Q=2.15 h$. $Q$

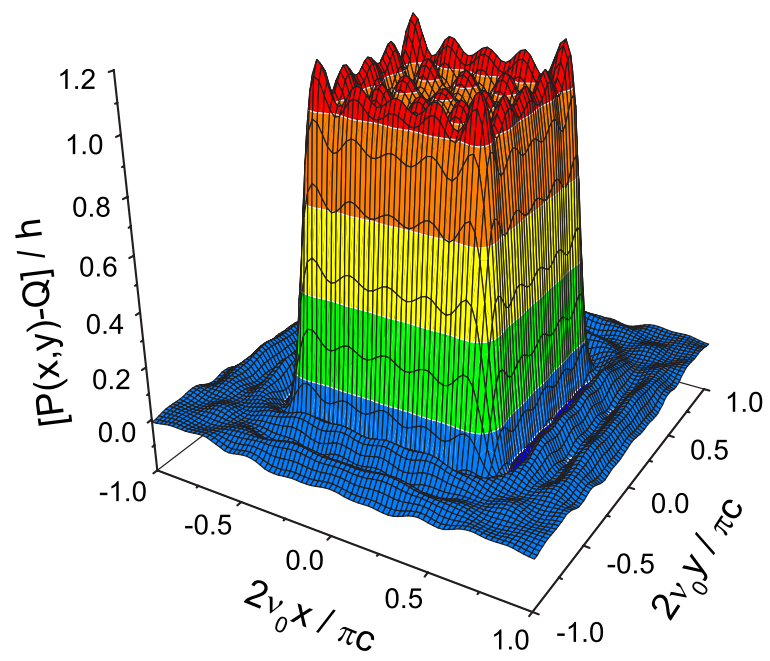

FIG. 2. (Color online) Approximation of a 2D pattern using direction variation and multiple exposures. Here the penalty deposition is already subtracted to get the truncated Fourier series. The upper limit is $N_{\max }=10$. and $h$ are in arbitrary units. This unit has to be chosen carefully to ensure the photoresist threshold dose falls between $Q$ and $Q+h$ [14]. For the places with exposure dose close to $Q$, the photoresist only has a small loss after the development. While for the places with exposure dose close to $Q+h$, the photoresist is completely removed.

We also use this method to simulate a practical pattern "TAMU," as shown in Fig. 3. For $N_{\max }=10$ the result has a lot of fluctuations and the average height of the characters is only half of the expected value $h$. The reason is that now the smallest angle width for this pattern is $\frac{2 \nu_{0} \Delta x}{c} \approx \frac{\pi}{20}, 20$ times

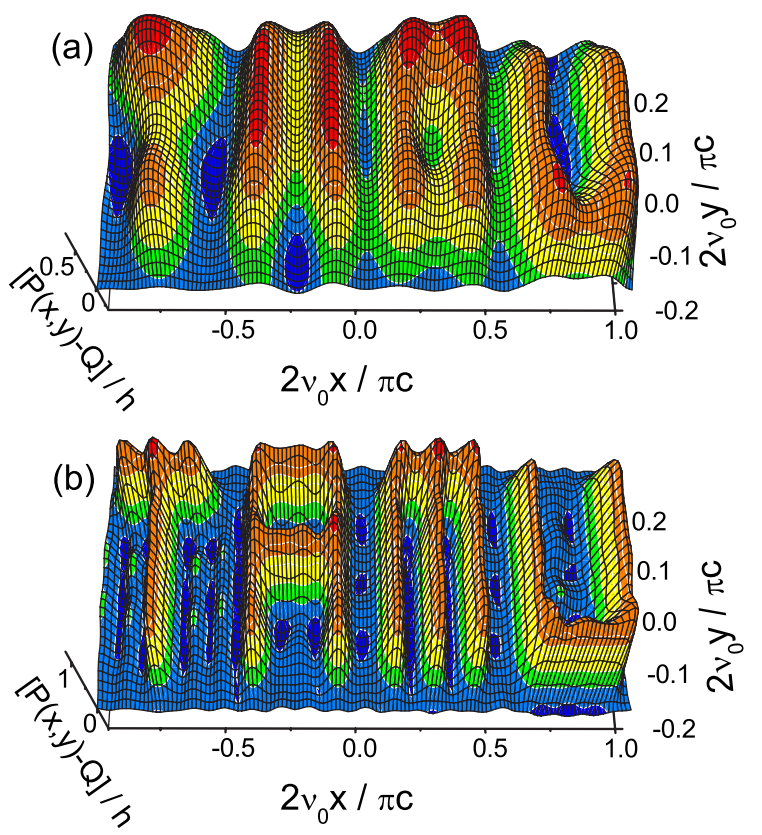

FIG. 3. (Color online) Approximation of the 2D pattern "TAMU" with a different number of components. (a) $N_{\max }=10, Q$ $=1.68 \mathrm{~h}$. (b) $N_{\max }=20, Q=4.25 \mathrm{~h}$. 
smaller than the angle width of the previous square pattern. We cannot increase this width because that will make the whole range of the pattern larger than $2 \pi$ and overlap with the other periods. Such a small angle width requires higher order components in the summation to obtain a good simulation. As one can see in Fig. 3(b), for $N_{\max }=20$ the characters are clearly defined and the average height is around $h$. However, the penalty deposition is larger in this case since we include more terms.

\section{DISCUSSION AND CONCLUSION}

Compared with the generalized method of quantum lithography [15], we require neither superposition of entangled Fock states nor broadband sensitive substrate. In addition, instead of a few 2D patterns, our method can approximate any $2 \mathrm{D}$ pattern in principle. Compared with the other interferometric schemes which use $N$-photon absorbers, they require the signal frequency summation equal to the level separation of the substrate, while in our scheme the summation can be much larger than the level separation. We can always get a larger summation by increasing $\nu_{n \pm}$ or add another signal field, only limited by the requirement of the bandwidth. So we can have higher fundamental frequency which means a smaller pattern, and more Fourier components which means better simulation quality. This makes our scheme very suitable to simulate an arbitrary pattern at the subwavelength scale. Another advantage is that we can achieve a certain spatial frequency by either varying the field frequencies or varying the incidence angles, which provides more freedom for adjusting.

In summary, we extend our previous method [12] to generate arbitrary $1 \mathrm{D}$ or $2 \mathrm{D}$ patterns. We modify the resonance condition so that the fundamental frequency $\nu_{0}$ can be much larger than the signal frequencies, leading to subwavelength resolution. With suitable frequency summations and directions we make multiple exposures, which correspond to a truncated Fourier series that can simulate any pattern in principle. This pattern will repeat in two dimensions as a result of interferometric lithography. We also show that the smallest angle width of the pattern decides the number of components needed in the truncated Fourier series.

For implementation of this scheme, the constraint of the bandwidth is not a major problem as pointed out in [12]; but a substrate with high multiphoton efficiencies may not be easy to find. To achieve fringes for different $N$, we have to choose the signal fields carefully according to the level structure of the substrate atoms. Still the choice is flexible since we can change the number, frequencies, and incident angles of the signal fields, and the drive frequency as well. The transverse mode profiles of the incident waves can be a source of imperfection. However, this may not be a problem if the size of the pattern is small.

\section{ACKNOWLEDGMENT}

We gratefully acknowledge the support from the Office of Naval Research, the Air Force Office of Scientific Research, and DARPA-QuIST.
[1] S. R. J. Brueck, S. H. Zaidi, X. Chen, and Z. Zhang, Microelectron. Eng. 41/42, 145 (1998).

[2] Lord Rayleigh, Philos. Mag. 8, 261 (1879).

[3] G. L.-T. Chiu and J. M. Shaw, IBM J. Res. Dev. 41, 3 (1997).

[4] E. Yablonovitch and R. B. Vrijen, Opt. Eng. 38, 334 (1999).

[5] M. M. Alkaisi, R. J. Blaikie, S. J. McNab, R. Cheung, and D. R. S. Cumming, Appl. Phys. Lett. 75, 3560 (1999).

[6] A. N. Boto, P. Kok, D. S. Abrams, S. L. Braunstein, C. P. Williams, and J. P. Dowling, Phys. Rev. Lett. 85, 2733 (2000).

[7] S. Kawata, H.-B. Sun, T. Tanaka, and K. Takada, Nature (London) 412, 697 (2001).

[8] M. D’Angelo, M. V. Chekhova, and Y. Shih, Phys. Rev. Lett. 87, 013602 (2001).
[9] A. Pe'er, B. Dayan, M. Vucelja, Y. Silberberg, and A. A. Friesem, Opt. Express 12, 6600 (2004).

[10] S. J. Bentley and R. W. Boyd, Opt. Express 12, 5735 (2004).

[11] G. Khoury, H. S. Eisenberg, E. J. S. Fonseca, and D. Bouwmeester, Phys. Rev. Lett. 96, 203601 (2006).

[12] P. R. Hemmer, A. Muthukrishnan, M. O. Scully, and M. S. Zubairy, Phys. Rev. Lett. 96, 163603 (2006).

[13] A. Cho, Science 312, 672 (2006).

[14] Harry J. Levinson, Principles of Lithography (SPIE, Bellingham, WA, 2001).

[15] P. Kok, A. N. Boto, D. S. Abrams, C. P. Williams, S. L. Braunstein, and J. P. Dowling, Phys. Rev. A 63, 063407 (2001). 